\title{
A HILBERT TYPE INEQUALITY
}

\author{
G. D. HANDLEY, J. J. KOLIHA AND J. PEČARIĆ
}

\begin{abstract}
In this paper we obtain a new inequality of Hilbert type for a finite number of nonnegative sequences of real numbers from which we can recover as a special case an inequality due to Pachpatte. We also obtain an integral variant of the inequality.
\end{abstract}

\section{Introduction}

The well known Hilbert's inequality [2, p.226] has been generalized in many directions by a number of mathematicians (see $[1,2,3,4,5]$ ). The purpose of the present paper is to derive a new inequality of Hilbert type, which will subsume, as a special case, a recent result of Pachpatte [7, Theorem 1].

Theorem 1. Let $\left\{a_{i, m_{i}}\right\}(i=1, \ldots, n)$ be $n$ sequences of nonnegative real numbers defined for $m_{i}=1,2, \ldots, k_{i}$ with $a_{1,0}=a_{2,0}=\cdots=a_{n, 0}=0$ and let $\left\{p_{i, m_{i}}\right\}$ be $n$ sequences of positive real numbers defined for $m_{i}=1,2, \ldots, k_{i}$, where $k_{i}(i=1,2, \ldots, n)$ are natural numbers. Set $P_{i, m_{i}}=\sum_{s=1}^{m_{i}} p_{i, s}(i=1, \ldots, n)$. Let $\phi_{i}(i=1, \ldots, n)$ be $n$ real-valued nonnegative convex and submultiplicative functions defined on $\mathbb{R}_{+}=[0, \infty)$, let $\alpha_{i} \in(0,1)$, and set $\alpha_{i}^{\prime}=1-\alpha_{i}(i=1, \ldots, n), \alpha=\sum_{i=1}^{n} \alpha_{i}$ and $\alpha^{\prime}=\sum_{i=1}^{n} \alpha_{i}^{\prime}=n-\alpha$. Then

$$
\begin{aligned}
& \sum_{m_{1}=1}^{k_{1}} \ldots \sum_{m_{n}=1}^{k_{n}} \frac{\prod_{i=1}^{n} \phi_{i}\left(a_{i, m_{i}}\right)}{\left(\sum_{i=1}^{n} \alpha_{i}^{\prime} m_{i}\right)^{\alpha^{\prime}}} \\
\leq & M\left(k_{1}, \ldots, k_{n}\right) \prod_{i=1}^{n}\left\{\sum_{m_{i}=1}^{k_{i}}\left(k_{i}-m_{i}+1\right)\left[p_{i, m_{i}} \phi_{i}\left(\frac{\nabla a_{i, m_{i}}}{p_{i, m_{i}}}\right)\right]^{1 / \alpha_{i}}\right\}^{\alpha_{i}},
\end{aligned}
$$

where

$$
M\left(k_{1}, \ldots, k_{n}\right)=\frac{1}{\left(\alpha^{\prime}\right)^{\alpha^{\prime}}} \prod_{i=1}^{n}\left(\sum_{m_{i}=1}^{k_{i}}\left[\frac{\phi_{i}\left(P_{i, m_{i}}\right)}{P_{i, m_{i}}}\right]^{1 / \alpha_{i}^{\prime}}\right)^{\alpha_{i}^{\prime}}
$$

and

$$
\nabla a_{i, m_{i}}=a_{i, m_{i}}-a_{i, m_{i}-1} \quad(i=1, \ldots, n) .
$$

Received March 30, 2000.

2000 Mathematics Subject Classification. 26D10, 26D15.

Key words and phrases. Hilbert type inequality, Hölder inequality, Jensen's inequality. 
Proof. From the hypotheses it is easy to observe that

$$
a_{i, m_{i}}=\sum_{s_{i}=1}^{m_{i}} \nabla a_{i, s_{i}} \quad\left(m_{i}=1,2, \ldots, k_{i}, i=1, \ldots, n\right) .
$$

So we have

$$
\begin{aligned}
\phi_{i}\left(a_{i, m_{i}}\right) & =\phi_{i}\left\{\frac{P_{i, m_{i}} \sum_{s_{i}=1}^{m_{i}} p_{i, s_{i}}\left(\nabla a_{i, s_{i}} / p_{i, s_{i}}\right)}{\sum_{s_{i}=1}^{m_{i}} p_{i, s_{i}}}\right\} \\
& \leq \phi_{i}\left(P_{i, m_{i}}\right) \phi_{i}\left\{\frac{\sum_{s_{i}=1}^{m_{i}} p_{i, s_{i}}\left(\nabla a_{i, s_{i}} / p_{i, s_{i}}\right)}{\sum_{s_{i}=1}^{m_{i}} p_{i, s_{i}}}\right\} \\
& \leq \phi_{i}\left(P_{i, m_{i}}\right) \frac{\sum_{s_{i}=1}^{m_{i}} p_{i, s_{i}} \phi_{i}\left(\nabla a_{i, s_{i}} / p_{i, s_{i}}\right)}{P_{i, m_{i}}}
\end{aligned}
$$

for $i=1, \ldots, n$.

Further, by Hölder's inequality (see [6, p. 99]) we have

$$
\begin{aligned}
\prod_{i=1}^{n} \phi_{i}\left(a_{i, m_{i}}\right) & \leq \prod_{i=1}^{n}\left(\frac{\phi_{i}\left(P_{i, m_{i}}\right)}{P_{i, m_{i}}} \sum_{s_{i}=1}^{m_{i}} p_{i, s_{i}} \phi_{i}\left(\nabla a_{i, s_{i}} / p_{i, s_{i}}\right)\right) \\
& \leq \prod_{i=1}^{n}\left\{\left[\frac{\phi_{i}\left(P_{i, m_{i}}\right)}{P_{i, m_{i}}}\right]\left[m_{i}\right]^{\alpha_{i}^{\prime}}\left[\sum_{s_{i}=1}^{m_{i}}\left(p_{i, s_{i}} \phi_{i}\left(\nabla a_{i, s_{i}} / p_{i, s_{i}}\right)^{1 / \alpha_{i}}\right]^{\alpha_{i}}\right\} .\right.
\end{aligned}
$$

Let us note that

$$
\left(\prod_{i=1}^{n}\left(m_{i}\right)^{\alpha_{i}^{\prime}}\right)^{1 / \alpha^{\prime}} \leq \frac{1}{\alpha^{\prime}} \sum_{i=1}^{n} \alpha_{i}^{\prime} m_{i}
$$

so we have

$$
\prod_{i=1}^{n}\left(m_{i}\right)^{\alpha_{i}^{\prime}} \leq \frac{1}{\left(\alpha^{\prime}\right)^{\alpha^{\prime}}}\left(\sum_{i=1}^{n} \alpha_{i}^{\prime} m_{i}\right)^{\alpha^{\prime}}
$$

and (2) becomes

$$
\prod_{i=1}^{n} \phi_{i}\left(a_{i m_{i}}\right) \leq \frac{\left(\sum_{i=1}^{n} \alpha_{i}^{\prime} m_{i}\right)^{\alpha^{\prime}}}{\left(\alpha^{\prime}\right)^{\alpha^{\prime}}} \prod_{i=1}^{n}\left\{\left[\frac{\phi_{i}\left(P_{i, m_{i}}\right)}{P_{i, m_{i}}}\right]\left[\sum_{s_{i}=1}^{m_{i}}\left[p_{i, s_{i}} \phi_{i}\left(\nabla a_{i, s_{i}} \phi_{i}\left(\nabla a_{i, s_{i}} / p_{i, s_{i}}\right)\right]^{1 / \alpha_{i}}\right]^{\alpha_{i}}\right\} .\right.
$$

Dividing both sides of (3) by $\left(\sum_{i=1}^{n} \alpha_{i}^{\prime} m_{i}\right)^{\alpha^{\prime}}$ and taking the sum over $m_{i}(i=1, \ldots, n)$ from 1 to $k_{i}$, then using Hölder's inequality (see [6, p. 99]) and interchanging the order of summation, we observe that

$$
\sum_{m_{1}=1}^{k_{1}} \cdots \sum_{m_{n}=1}^{k_{n}} \frac{\prod_{i=1}^{n} \phi_{i}\left(a_{i, m_{i}}\right)}{\left(\sum_{i=1}^{n} \alpha_{i}^{\prime} m_{i}\right)^{\alpha^{\prime}}}
$$




$$
\begin{aligned}
& \leq \frac{1}{\left(\alpha^{\prime}\right)^{\alpha^{\prime}}} \prod_{i=1}^{n}\left\{\sum_{m_{i}=1}^{k_{i}}\left[\frac{\phi_{i}\left(P_{i, m_{i}}\right)}{P_{i, m_{i}}}\right]\left[\sum_{s_{i}=1}^{m_{i}}\left(p_{i, s_{i}} \phi_{i}\left(\nabla a_{i, s_{i}} \phi_{i}\left(\nabla a_{i, s_{i}} / p_{i, s_{i}}\right)\right)^{1 / \alpha_{i}}\right]^{\alpha_{i}}\right\}\right. \\
& \leq \frac{1}{\left(\alpha^{\prime}\right)^{\alpha^{\prime}}} \prod_{i=1}^{n}\left\{\left[\sum_{m_{i}=1}^{k_{i}}\left[\frac{\phi_{i}\left(P_{i, m_{i}}\right)}{P_{i, m_{i}}}\right]^{1 / \alpha_{i}^{\prime}}\right]^{\alpha_{i}^{\prime}}\left[\sum_{m_{i}=1}^{k_{i}}\left[\sum_{s_{i}=1}^{m_{i}}\left(p_{i, s_{i}} \phi_{i}\left(\nabla a_{i, s_{i}} / p_{i, s_{i}}\right)\right)^{1 / \alpha_{i}}\right]\right]^{\alpha_{i}}\right\} \\
& =M\left(k_{1}, \ldots, k_{n}\right) \prod_{i=1}^{n}\left\{\sum_{m_{i}=1}^{k_{i}}\left[\sum_{s_{i}=1}^{m_{i}}\left(p_{i, s_{i}} \phi_{i}\left(\nabla a_{i, s_{i}} / p_{i, s_{i}}\right)\right)^{1 / \alpha_{i}}\right]\right\}^{\alpha_{i}} \\
& =M\left(k_{1}, \ldots, k_{n}\right) \prod_{i=1}^{n}\left\{\sum_{s_{i}=1}^{m_{i}}\left[p_{i, s_{i}} \phi_{i}\left(\nabla a_{i, s_{i}} / p_{i, s_{i}}\right)\right]^{1 / \alpha_{i}}\left(\sum_{m_{i}=s_{i}}^{k_{i}} i\right)\right\}^{\alpha_{i}} \\
& =M\left(k_{1}, \ldots, k_{n}\right) \prod_{i=1}^{n}\left\{\sum_{s_{i}=1}^{m_{i}}\left(k_{i}-s_{i}+1\right)\left[p_{i, s_{i}} \phi_{i}\left(\nabla a_{i, s_{i}} / p_{i, s_{i}}\right)\right]^{1 / \alpha_{i}}\right\}^{\alpha_{i}},
\end{aligned}
$$

which is equivalent to $(1)$.

Remark 2. For $\alpha_{1}=\cdots=\alpha_{n}=(n-1) / n$, (1) becomes

$$
\begin{aligned}
& \sum_{m_{1}=1}^{k_{1}} \cdots \sum_{m_{n}=1}^{k_{n}} \frac{\prod_{i=1}^{n} \phi_{i}\left(a_{i, m_{i}}\right)}{m_{1}+\cdots+m_{n}} \\
& \leq \bar{M}\left(k_{1}, \ldots, k_{n}\right) \prod_{i=1}^{n}\left\{\sum_{m_{i}=1}^{k_{i}}\left(k_{i}-m_{i}+1\right)\left[p_{i, m_{i}} \phi_{i}\left(\frac{\nabla a_{i, m_{i}}}{p_{i, m_{i}}}\right)\right]^{n /(n-1)}\right\}^{(n-1) / n},
\end{aligned}
$$

where

$$
\bar{M}\left(k_{1}, \ldots, k_{n}\right)=\frac{1}{n} \prod_{i=1}^{n}\left\{\sum_{m_{i}=1}^{k_{i}}\left[\frac{\phi_{i}\left(P_{i, m_{i}}\right)}{P_{i, m_{i}}}\right]^{n}\right\}^{1 / n} .
$$

For $n=2$, this is Pachpatte's result [7, Theorem 1].

There is also an integral analogue of Theorem 1.

Theorem 3. Let $f_{i} \in C^{1}\left[\left[0, k_{i}\right], \mathbb{R}_{+}\right], i=1, \ldots, n$, with $f_{i}(0)=0(i=1, \ldots, n)$, let $p_{i}\left(\sigma_{i}\right)$ be $n$ positive functions defined for $\sigma_{i} \in\left[0, x_{i}\right](i=1, \ldots, n)$, and set $P_{i}\left(s_{i}\right)=$ $\int_{0}^{s_{i}} p_{i}\left(\sigma_{i}\right) d \sigma_{i}$ for $s_{i} \in\left[0, x_{i}\right]$ where $x_{i}$ are positive real numbers. Let $\phi_{i}, \alpha_{i}, \alpha_{i}^{\prime}, \alpha$ and $\alpha^{\prime}$ be as in Theorem 1. Then

$$
\begin{aligned}
& \int_{0}^{x_{1}} \cdots \int_{0}^{x_{n}} \frac{\prod_{i=1}^{n} \phi_{i}\left(f_{i}\left(s_{i}\right)\right)}{\left(\sum_{i=1}^{n} \alpha_{i}^{\prime} s_{i}\right)^{\alpha^{\prime}}} \\
\leq & L\left(x_{1}, \ldots, x_{n}\right) \prod_{i=1}^{n}\left\{\int_{0}^{x_{i}}\left(x_{i}-s_{i}\right)\left[p_{i}\left(s_{i}\right) \phi_{i}\left(f_{i}^{\prime}\left(s_{i}\right) / p_{i}\left(s_{i}\right)\right)\right]^{1 / \alpha_{i}} d s_{i}\right\}^{\alpha_{i}},
\end{aligned}
$$

where

$$
L\left(x_{1}, \ldots, x_{n}\right)=\frac{1}{\left(\alpha^{\prime}\right)^{\alpha^{\prime}}} \prod_{i=1}^{n}\left\{\int_{0}^{x_{i}}\left[\frac{\phi_{i}\left(P_{i}\left(s_{i}\right)\right.}{P_{i}\left(s_{i}\right)}\right]^{1 / \alpha_{i}^{\prime}} d s_{i}\right\}^{\alpha_{i}^{\prime}}
$$


Proof. From the hypotheses we have

$$
f_{i}\left(s_{i}\right)=\int_{0}^{s_{i}} f^{\prime}\left(\sigma_{i}\right) d \sigma_{i}, \quad s_{i} \in\left[0, x_{i}\right]
$$

Using Jensen's integral inequality (see $[6$, p. 6]), we obtain

$$
\begin{aligned}
\phi_{i}\left(f_{i}\left(s_{i}\right)\right) & =\phi_{i}\left\{\frac{P_{i}\left(s_{i}\right) \int_{0}^{s_{i}} p_{i}\left(\sigma_{i}\right)\left(f_{i}^{\prime}\left(\sigma_{i}\right) / p_{i}\left(\sigma_{i}\right)\right)}{\int_{0}^{s_{i}} p_{i}\left(\sigma_{i}\right) d \sigma_{i}} d \sigma_{i}\right\} \\
& \leq \phi_{i}\left(P_{i}\left(s_{i}\right)\right) \phi_{i}\left\{\frac{\int_{0}^{s_{i}} p_{i}\left(\sigma_{i}\right)\left(f_{i}^{\prime}\left(\sigma_{i}\right) / p_{i}\left(\sigma_{i}\right)\right) d \sigma_{i}}{\int_{0}^{s_{i}} p_{i}\left(\sigma_{i}\right) d \sigma_{i}}\right\} \\
& \leq \frac{\phi_{i}\left(P_{i}\left(s_{i}\right)\right)}{P_{i}\left(s_{i}\right)} \int_{0}^{s_{i}} p_{i}\left(\sigma_{i}\right) \phi_{i}\left(f_{i}^{\prime}\left(\sigma_{i}\right) / p_{i}\left(\sigma_{i}\right)\right) d \sigma_{i}, \quad i=1, \ldots, n .
\end{aligned}
$$

The rest of the proof is similar to that for Theorem 1.

Remark 4. For $\alpha_{1}=\cdots=\alpha_{n}=(n-1) / n$, (4) becomes

$$
\begin{aligned}
& \int_{0}^{x_{1}} \ldots \int_{0}^{x_{n}} \frac{\prod_{i=1}^{n} \phi_{i}\left(f_{i}\left(s_{i}\right)\right)}{s_{1}+\cdots+s_{n}} d s_{1} \cdots d s_{n} \\
\leq & \bar{L}\left(x_{1}, \ldots, x_{n}\right) \prod_{i=1}^{n}\left\{\int_{0}^{x_{i}}\left(x_{i}-s_{i}\right)\left[p_{i}\left(s_{i}\right) \phi_{i}\left(\frac{f_{i}^{\prime}\left(s_{i}\right)}{p_{i}\left(s_{i}\right)}\right)\right]^{n /(n-1)} d s_{i}\right\}^{(n-1) / n}
\end{aligned}
$$

where

$$
\bar{L}\left(x_{1}, \ldots, x_{n}\right)=\frac{1}{n} \prod_{i=1}^{n}\left\{\int_{0}^{x_{i}}\left[\frac{\phi_{i}\left(P_{i}\left(s_{i}\right)\right)}{P_{i}\left(s_{i}\right)}\right]^{n} d s_{i}\right\}^{1 / n}
$$

For $n=2$ we recover Pachpatte's result [7, Theorem 2].

\section{References}

[1] Y. C. Chow, On inequalities of Hilbert and Widder, J. London Math. Soc., 14(1939), 151-154.

[2] G. H. Hardy, J. E. Littlewood and G. Polya, Inequalities, Cambridge University Press, 1934.

[3] M. Gao, An improvement of the Hardy-Riesz extension of the Hilbert inequality, J. Math. Res. Exp., 14(1994), 255-259.

[4] M. Gao, On Hilbert's inequality and its applications, J. Math. Anal. Appl., 212(1997), 316-323.

[5] D. S. Mitrinović and J. E. Perčarić, On inequalities of Hilbert and Widder, Proc. Edinburgh Math. Soc., 34(1991), 411-414.

[6] D. S. Mitrinović, J. E. Pečarić and A. M. Fink, Classical and New Inequalities in Analysis, Kluwer Acad. Publ., Dordrecht, 1993.

[7] B. G. Pachpatte, A note on Hilbert type inequality, Tamkang J. Math., 29(1998), 293-298. 
Department of Mathematics and Statistics, University of Melbourne, VIC 3010, Australia. Department of Mathematics and Statistics, University of Melbourne, VIC 3010, Australia. Faculty of Textile Technology, University of Zagreb, 10000 Zagreb, Croatia. 